\title{
BMJ Open Central themes, core concepts and knowledge gaps concerning social media use, and mental health and well- being among adolescents: a protocol of a scoping review of published literature
}

\author{
Viktor Schønning (D) , ${ }^{1}$ Leif Edvard Aarø, ${ }^{1}$ Jens Christoffer Skogen ${ }^{1,2,3}$
}

To cite: Schønning V, Aarø LE, Skogen JC. Central themes, core concepts and knowledge gaps concerning social media use, and mental health and well-being among adolescents: a protocol of a scoping review of published literature. BMJ Open 2020;10:e031105. doi:10.1136/ bmjopen-2019-031105

- Prepublication history for this paper is available online. To view these files, please visit the journal online (http://dx.doi. org/10.1136/bmjopen-2019031105).

Received 16 April 2019

Revised 21 December 2019

Accepted 23 December 2019

Check for updates

(c) Author(s) (or their employer(s)) 2020. Re-use permitted under CC BY-NC. No commercial re-use. See rights and permissions. Published by BMJ.

${ }^{1}$ Department of Health Promotion, Norwegian Institute of Public Health, Bergen, Norway ${ }^{2}$ Faculty of Health Sciences, University of Stavanger,

Stavanger, Norway

${ }^{3}$ Alcohol and Drug Research

Western Norway (KoRFor),

Stavanger University Hospital,

Stavanger, Norway

Correspondence to

Viktor Schønning;

Viktor.schonning@fhi.no

\section{ABSTRACT}

Introduction The use of social media has risen steadily since its introduction in the early 2000 s, and today there are between 2 and 3 billion users worldwide. Research on the link between use of social media and mental health has resulted in a vast number of studies covering diverse aspects of the link between them. The existing body of knowledge on use of social media, and mental health and well-being among adolescents is complex and difficult to follow. In this paper, we present a protocol for a scoping review to systematically identify and summarise the central research foci and knowledge gaps in the research field of social media use, and mental health and well-being among adolescents.

Methods and analysis The current scoping review will adhere to the Preferred Reporting Items for Systematic Review and Meta-Analyses extension for Scoping Reviews. The first step is to search relevant databases for eligible studies. Relevant databases are CINAHL, Ovid Medline, Embase, PsycINF0, Sociological Abstracts, Sociological Services Abstracts, ERIC, Cochrane Database of Systematic Reviews, CRD (Database of Abstracts of Reviews of Effects), NHS EED, HTA and Epistemonikos. Next, two reviewers from the research team will independently screen the identified studies for eligibility. Data extraction and data synthesis will be performed and result in summarised themes based on the findings. Ethics and dissemination A scoping review can be described as a method of gaining an overview and understanding of a research area, with its strengths and weaknesses, and as it involves peer-reviewed and published articles, a scoping review does not require ethical approval. We expect that the results from the current scoping review will produce a consolidated overview of existing studies and research gaps, and gather this knowledge into a coherent review. The results will be disseminated through relevant journals and conferences.

\section{BACKGROUND}

Social media is a relatively new phenomenon with an increasing popularity. The number of social media users worldwide has increased rapidly in the last years, reaching 2 billion in 2015 and is estimated to reach 3 billion users
Strengths and limitations of this study

- A strength of the current study is that it will be one of the first scoping reviews to systematically identify and summarise the central research foci and knowledge gaps in the research field of social media use, and mental health and well-being among adolescents (13-19 years) in both qualitative and quantitative studies.

- Another strength is that the search strategy includes several electronic databases with published peerreviewed literature, with an aim to cover all relevant research publications.

- Initial selection of articles will be done by two reviewers independently which is considered a strength of the study.

- It is considered a strength that data extraction from included articles will be done by two reviewers independently to ensure the quality of the collected information.

- Being a scoping review, no formal assessment of study quality will be carried out. This is considered a limitation of the current study.

in 2021. ${ }^{1}$ Among youth aged $12-15$ years in the UK, $99 \%$ go online during a week, averaging 20 hours per week and $69 \%$ have a social media profile according to a report on media use. $^{2}$ Today, social media use is ubiquitous in adolescents worldwide regardless of differences such as culture, geographic region or socioeconomic status. Social media includes services such as Facebook, Twitter, Snapchat and Instagram, with Facebook being the largest social media based on the number of users, followed by YouTube and WhatsApp. ${ }^{34}$ Young adults are more likely than older adults to use social media, with the demographic of users varying slightly between the type of media. ${ }^{5}$ The effects of increased social media use on youth health are still largely unknown, though some studies indicate detrimental 
effects on mental health. ${ }^{4}$ A US survey from 2016 found a significant association between social media use and increased depression, but the mechanisms and direction of the association were not identified. ${ }^{6}$ A systematic narrative review reports contradictory findings with both beneficial and harmful effects of social media use. ${ }^{7}$ There is currently an ongoing debate regarding the strength of the association between adolescent well-being and the use of digital technology and social media with Twenge and colleagues being adamant about its negative impact. ${ }^{8}$ On the other side, Orben and Przybylski have found that the association between digital technology use and adolescent well-being is negative but only explaining $0.4 \%$ of the variation in well-being, and they suggest that these effects are too small to warrant any policy change. ${ }^{9}$ The possibility that concerns regarding social media use may be exaggerated is also suggested by Berryman and colleagues in their study from $2018 .{ }^{10}$ There is also the possibility that the issue of moral panic is contributing to misrepresenting the detrimental effects of social media use on adolescents well-being. Moral panic can be defined as an exaggerated concern about a supposed threat to the current culture, way of life or society's current structure. It is a phenomenon which has been heavily discussed regarding the effects of introducing new media technologies such as video games, TV and radio. ${ }^{11}$ It is, however, difficult to pinpoint if moral panic exists and what role it plays in presenting the evidence of social media use on well-being. Given the research interest in the link between adolescents' social media use, and mental health and well-being, the planned scoping review will establish an overview over the existing body of knowledge and contribute to advance this field of research. Given the recency of the phenomenon, it is vital to identify and describe core themes as well as knowledge gaps when it comes to the effect of social media use on adolescent's mental health and well-being. The realm of social media is complex and multi-layered with several stakeholders and a constantly changing technological landscape. The content of social media is both user generated and commercially generated and there are often both corporate and public interests and stakeholders in the phenomenon. A scoping review would help provide a foundation for further research, which in time will provide a knowledge base for policymaking and service delivery.

The purpose of scoping reviews can be described as a way of mapping 'the key concepts underpinning a research area, and the main sources and types of evidence available, and can be undertaken as standalone projects in their own right, especially where an area is complex or has not been reviewed comprehensively before'. ${ }^{2}$ In our context, a scoping review will help provide an understanding of the 'big picture' and the main foci of research within the field of social media and mental health and well-being among adolescents, as well as the data sources and research instruments typically used. By using the scoping review approach, the current study aims to produce a consolidated overview of studies with diverging methodological designs and gather this knowledge into a coherent review. Furthermore, one of the most frequent reasons for conducting a scoping review is to identify gaps in the research literature. ${ }^{13}$ A vast number of studies on social media use and mental health has been conducted over the last decade, but to the authors' knowledge no scoping review has yet been carried out.

The review described here will follow the framework put forward by Arksey and O'Malley, which can be described in five steps: (1) identifying the research question, (2) identifying relevant studies, (3) selecting studies, (4) charting the data and (5) collating, summarising and reporting the results. ${ }^{13}$

\section{AIMS}

This scoping review aims to give an overview of the main research questions that have been focused on in relation to use of social media among adolescents and mental health and well-being. Both quantitative and qualitative studies are of interest. Three specific secondary research questions will be addressed and together with the main research question serve as a template for organising the results:

- Which aspects of mental health and well-being have been the focus or foci of research so far?

- Has the research focused on different research aims across gender, ethnicity, socioeconomic status, geographic location? What kind of findings are reported across these groups?

-What are the main sources of evidence related to social media that have been used in the studies identified?

\section{DEFINING ADOLESCENCE AND SOCIAL MEDIA}

In the present review, adolescence is defined as those between 13 and 19 years of age. We chose the age of 13 as our lower limit as nearly all social media services require users to be at least 13 years of age to access and use their services. ${ }^{14}$ This includes Facebook, Snapchat, Twitter, Instagram and Skype. ${ }^{14}$ All pertinent studies which present results relevant for this age range are within the scope of this review. Social media is a broad term that is difficult to pin down. We have chosen to use the following definition offered by Kietzmann and colleagues (p1): 'Social media employ mobile and web-based technologies to create highly interactive platforms via which individuals and communities share, co-create, discuss, and modify user-generated content'. ${ }^{15}$ This definition can be applied to Kaplan and Haenlein's ${ }^{16}$ classification scheme that specify types of social media across two axes-social presence/media richness and self-presentation/self-disclosure (see table 1).

The chosen definition of social media excludes other use of electronic or web-based media or programmes, such as medical or health-related services (eg, monitoring devices or medical reference), one-way transmissions of content (eg, podcasts) and real-time exchanges via technology (eg, Skype). We also exclude joint projects involving via technology (eg, Microsoft Whiteboard). Online discussion 
Table 1 Characteristics of social media across the axes: social presence/media richness and self-presentation/selfdisclosure. Adapted from Kaplan and Haenlein ${ }^{16}$

\begin{tabular}{|c|c|c|c|c|}
\hline & & \multicolumn{3}{|c|}{ Social presence/media richness } \\
\hline & & Lower & Medium & Higher \\
\hline \multirow[t]{2}{*}{$\begin{array}{l}\text { Self-presentation/self- } \\
\text { disclosure }\end{array}$} & High & Blogs & $\begin{array}{l}\text { Vlogs/social networking sites (eg, } \\
\text { Facebook) }\end{array}$ & $\begin{array}{l}\text { Virtual social worlds (eg, Second } \\
\text { Life) }\end{array}$ \\
\hline & Low & $\begin{array}{l}\text { Collaborative } \\
\text { projects (eg, } \\
\text { Wikipedia) }\end{array}$ & $\begin{array}{l}\text { Content communities (eg, Twitch, } \\
\text { YouTube) }\end{array}$ & $\begin{array}{l}\text { Virtual game worlds (eg, League } \\
\text { of Legends, Apex Legends) }\end{array}$ \\
\hline
\end{tabular}

forums and bulletin boards will however be included, as the content is at least partly generated by the users.

\section{DATA SOURCES AND SEARCH STRATEGY}

The following data sources were chosen due to their scope that include social sciences and public health research: CINAHL, Ovid Medline, Embase, PsycINFO, Sociological Abstracts, Sociological Services Abstracts, ERIC, Cochrane Database of Systematic Reviews, CRD (Database of Abstracts of Reviews of Effects), NHS EED, HTA and Epistemonikos. The search terms included combinations of different variants of 'adolescent', 'mental health', 'well-being' and 'social media', and only studies published in the last 5 years were eligible. This limited search period was chosen mainly due to rapid changes in the use and types of use of social media. Findings more than 5 years old were therefore deemed to be less relevant to shed light on our research questions. There were also practical reasons to limiting our search to 2014, related to available resources. Before starting the full search, the search strategy was piloted in order to assess relevance. The start date for the search was April 2019, with additional searches in May 2019. The snowballing technique will be used to identify articles that are not covered by our search, but only for studies published after 2014 . $^{17}$ Publisher and journals will be assessed for legitimacy according to the relevant criteria described by the Directory of Open Access Journals (for open access publications) and other relevant sources. ${ }^{18}$

\section{STUDY SELECTION: EXCLUSION AND INCLUSION CRITERIA Inclusion}

- Published in English.

- Peer-reviewed papers.

- Published within the last 5 years (since 2014).

- Participants/informants aged between 13 and 19 years.

- Empirical studies of primary research.

- Observational or participatory studies.

- Systematic reviews (and meta-analyses).

- Explicit focus on mental health, sleep and/or well-being.

- Explicit focus on social media.

\section{Exclusion}

- Editorials, opinion pieces, commentaries.

- Study or review protocols.

- Book chapters.

- Publications not peer-reviewed.

- Non-empirical studies.

- Theoretical studies, perspective articles.

- Specific (sub)populations such as individuals with chronic illness or physical or mental disabilities.

- Specific subpopulations, minority groups.

- Intervention studies.

- Treatment studies.

- Internet-based or app-based therapy.

- Unpublished studies/conference proceedings.

- Not within our definition of social media.

- Studies were social media is only used as a moderator.

- Studies which focus on internet gambling.

- Clinical studies or clinical case reports.

- Studies focusing on the use of online information.

- Studies focusing on aggression and violence (beyond cyber bullying).

- Studies focusing on brain disorders/cognitive disorders.

- Studies focusing on information processing, decisionmaking or personality.

The initial criteria used for study selection are part of an iterative process, ${ }^{19}$ and we will follow a two-stage approach:

1. Learning and adjusting stage: Two reviewers will independently screen titles and abstracts of 300 randomly drawn studies for eligibility. The purpose of this stage is to clarify the initial inclusion criteria, and to identify any uncertainties related to the inclusion and exclusion of papers. A third independent reviewer can be consulted if necessary. Adjustments will be made if deemed necessary.

2. Final selection stage: Two reviewers will independently screen titles and abstracts of all studies for eligibility according to the revised and updated inclusion and exclusion criteria. The full text of studies assessed as 'relevant' or 'unclear' will then be independently evaluated by two reviewers. The interrater agreement will be computed and discrepancies will be resolved by consensus or if necessary, by adjudication by a third independent reviewer. 
The selection process will be illustrated by a flowchart indicating the stages from unsorted search results to the total number of included studies. Study selection will be accomplished and organised using the Rayyan QCRI software (https://rayyan.qcri.org/welcome). Both reviewers are trained clinical psychologists based on the scientistpractitioner model.

\section{DATA EXTRACTION AND ORGANISATION}

Data extraction will be done for all included papers by one reviewer. A randomly drawn proportion (5\%-10\% depending on the total number of included papers) of these papers will also be assessed by a second reviewer to ensure adherence to the data extraction plan as well as assessment of the quality of the extraction. Data will be extracted according to the following details:

- Bibliographic information.

- Author information.

- Title.

- Journal.

- Year of publication.

- Country of corresponding author.

- Information about study design.

- Quantitative or qualitative.

- Study design.

- Study setting.

- Participants.

- Gender distribution.

- Subject matter information.

- Main aim of study.

- Type of social media use.

- How social media use was assessed.

- Mental health or well-being measure.

- Report gender differences.

- Main finding/conclusion (free text).

- Type of scales used (only applicable in quantitative studies).

Data extraction and synthesis will be accomplished and organised using an electronic data spreadsheet.

\section{DATA SYNTHESIS: QUANTIFICATION AND NARRATIVE APPROACH}

The extracted data will first be submitted to simple quantitative analysis using descriptive statistics (frequencies and measures of central tendencies) with purpose of providing an overview of the main characteristics of the included studies. ${ }^{12}$ The data will also be narratively assessed with a focus on the core themes and concepts emerging from the extracted data. This thematic analysis will be performed by two reviewers independently, guided by three main axes:

1. Mental health: Which aspects of mental health were focussed in each study?

2. Social media: What type of social media use was the focus of the study? Typology will be based on Kaplan and Haenlein's ${ }^{16}$ classification scheme.
3. Type of study: Was the study qualitative or quantitative?

The results will be compared and consolidated by consensus between the two reviewers. The resulting themes will be reviewed by a third independent reviewer to ensure validity and credibility. The themes will be reported to highlight the similarities, patterns and differences found in the literature, using a content-based approach. The reviewers are trained clinical psychologists educated based on the scientist-practitioner model. All but one of the researchers involved have experience with different kinds of reviews, such as narrative reviews and systematic reviews.

\section{PUBLIC AND PATIENT INVOLVEMENT}

No patient involved.

\section{PRESENTATION OF THE RESULTS}

The purpose of scoping reviews is to aggregate and synthesise data in order to gain an overview of a field of research. Our results will be presented in tables and visual illustrations (eg, graphs and figures) and according to the emerging themes from the analyses described above. The exact presentation format will be further specified as the review process develops. ${ }^{13}$ The current scoping review will strive to adhere to the Preferred Reporting Items for Systematic reviews and Meta-analyses extension for Scoping Reviews as presented by Tricco and colleagues. ${ }^{20}$

\section{ETHICAL CONSIDERATIONS AND DISSEMINATION OF KNOWLEDGE GAINED}

This protocol is a transparent description of the planned methodology for a scoping review. Our aspiration is that this protocol will lay the groundwork for a comprehensive and rigorous review that can contribute to the advancement of research related to adolescents, social media, mental health and well-being. The review will contribute to the advancement of research on this subject by identifying central research themes and gaps in knowledge and research. The results will be disseminated through publications as well as presentations at relevant conferences. Furthermore, our results may inform new research and policy initiatives addressing the subject matter.

Acknowledgements The authors would like to thank Bergen municipality, Hordaland County Council and Western Norway University of Applied Sciences for their collaboration and help with the review.

Contributors All authors, VS, LEA and JCS, have made substantive intellectual contributions to the development of this protocol. JCS conceptualised the review approach, provided general guidance to the research team and drafted the first version. VS developed the draft further, and all authors were involved in further revisions of the draft, review questions and the review design. All authors approved the final version of the manuscript.

Funding This review is partly funded by Regional Research Funds in Norway, funding \#RFF297031. No other specific funding was received for the present project. The present project is associated with a larger innovation-project lead by Bergen municipality in Western Norway related to the use of social media and mental health and well-being. The innovation project is funded by a programme 
initiated by the Norwegian Directorate of Health, and aims to explore social media as platform for health promotion among adolescents.

Competing interests None declared.

Patient consent for publication Not required.

Provenance and peer review Not commissioned; externally peer reviewed.

Open access This is an open access article distributed in accordance with the Creative Commons Attribution Non Commercial (CC BY-NC 4.0) license, which permits others to distribute, remix, adapt, build upon this work non-commercially, and license their derivative works on different terms, provided the original work is properly cited, appropriate credit is given, any changes made indicated, and the use is non-commercial. See: http://creativecommons.org/licenses/by-nc/4.0/.

\section{ORCID iD}

Viktor Schønning http://orcid.org/0000-0002-3616-4950

\section{REFERENCES}

1 eMarketer. Number of social media users worldwide from 2010 to 2021 (in billions), 2017. Available: https://www.statista.com/statistics/ 278414/number-of-worldwide-social-network-users/ [Accessed 21 Mar 2019].

2 Ofcom, Children and Parents: Media Use and Attitudes Report 2018

3 DataReportal. Most popular social networks worldwide as of January 2019, ranked by number of active users (in millions), 2019. Available: https://www.statista.com/statistics/272014/global-social-networksranked-by-number-of-users/ [Accessed 21 Mar 2019].

4 Han B. Social media burnout: definition, measurement instrument, and why we care. Journal of Computer Information Systems 2018;58:122-30.

5 Duggan M, Brenner J. The demographics of social media users 2012. Washington, DC: Pew Research Center's Internet \& American Life Project, 2013.

6 Lin LY, Sidani JE, Shensa A, et al. Association between social media use and depression among U.S. young adults. Depress Anxiety 2016;33:323-31.
7 Best P, Manktelow R, Taylor B. Online communication, social media and adolescent wellbeing: a systematic narrative review. Child Youth Serv Rev 2014;41:27-36.

8 Twenge JM, Campbell WK. Media use is linked to lower psychological well-being: evidence from three datasets. Psychiatr $Q$ 2019;90:311-31.

9 Orben A, Przybylski AK. The association between adolescent well-being and digital technology use. Nature Human Behaviour 2019;3:173-82.

10 Berryman C, Ferguson CJ, Negy C. Social media use and mental health among young adults. Psychiatr Q 2018;89:307-14.

11 Mueller M. Challenging the social media moral panic: preserving free expression under Hypertransparency. The CATO Papers on Public Policy, 2019

12 Mays N, Roberts E, Popay J. Synthesising research evidence, in Methods for studying the delivery and organisation of health services. In: Fulop N, ed. London: Routledge, 2001.

13 Arksey H, O'Malley L. Scoping studies: towards a methodological framework. Int J Soc Res Methodol 2005;8:19-32.

14 International, C. Age restrictions on social media services, 2018. Available: https://www.childnet.com/blog/age-restrictions-on-socialmedia-services [Accessed 30 Sep 2019].

15 Kietzmann JH, Hermkens K, McCarthy IP, et al. Social media? get serious! understanding the functional building blocks of social media. Bus Horiz 2011;54:241-51.

16 Kaplan AM, Haenlein M. Users of the world, unite! the challenges and opportunities of social media. Bus Horiz 2010;53:59-68.

17 Sayers A. Tips and tricks in performing a systematic review. Br J Gen Pract 2007:57:759.

18 Laine C, Winker MA. Identifying predatory or pseudo-journals. Biochem Med 2017;27:285-91.

19 Levac D, Colquhoun H, O'Brien KK. Scoping studies: advancing the methodology. Implement Sci 2010;5:69.

20 Tricco AC, Lillie E, Zarin W, et al. PRISMA extension for scoping reviews (PRISMA-ScR): checklist and explanation. Ann Intern Med 2018;169:467-73. 